\title{
SLE experience over unreliable network links
}

\author{
Ciprian Furtuna ${ }^{1}$ and Carla Garcia ${ }^{2}$ \\ LSE Space GmbH, Argelsrieder Feld 22, 82234, Wessling, Germany \\ Wilfried Kruse ${ }^{3}$ \\ Deutsches Zentrum für Luft und Raumfahrt (DLR), German Space Operations Centre(GSOC), \\ Oberpfaffenhofen,Münchner str 20, Wessling, 82234, Germany
}

\begin{abstract}
Despite the great advances of technology in networking areas, space missions sometimes face difficulties to achieve a successful operational state when high reliability data links are not available between their control centres and the ground stations, causing loss of data and disconnections. The reasons are varied, from time and cost constraints to design and implementation restrictions, as well as additional limitations imposed by the Mission Control System (MCS). The objective of this paper is to share the experience gained at different stages of past spacecraft missions where CCSDS Space Link Extension services were not offered in the initial design, to present the solutions found to mitigate the data losses and to illustrate the positive impact to the overall mission following the implementation of CCSDS SLE services. Comparisons between different ground protocols, used extensively in space operations, to transport telemetry at higher data rates in real time between remote ground stations and control centres, and appropriate parameter fine-tuning that led to improved data delivery reliability are taken into consideration as well. This paper presents the operational success of the deployment of the SLE protocol to overcome the difficulties caused by the specified restrictions and limitations. The result of this analysis, or just parts of it, could be useful to space agencies in planning and designing their future missions in an optimal manner with respect to the ground network design and costs.
\end{abstract}

\section{Introduction}

GSOC integrated the SLE transfer services into its ground data system in 2002 and has been using them up to the present time for almost all of its satellite missions. The normal process of a ground data system preparation for a given mission starts around two to three years before launch. For the mission in question, GSOC had to speed up the process and get ready to take over an already running mission in about half a year. That was the first challenge.

Even though GSOC has gained a lot of ground systems experience over the years working in the space business, each mission always presents special requirements and constraints on the ground segment that result into new challenges to overcome. This paper shows clear examples, where the following boundary conditions, among others, were in place:

- Use of an unreliable data link (via Internet) between the main ground station and the control centre

- MCS limitation of data processing/reception

- Initial design that did not use SLE services, but relied on the use of the Cortex protocol and the functionality of the baseband unit

- Wrong assumption/estimation of the bandwidth provided by the VPN data link

Besides that, an additional challenge was caused by the fact that there were no offline services available for this mission at our control centre, even though they were presented during the initial ground data system design for a different control centre. Of course under these circumstances real time data delivery and completeness played a very important role and became a high priority.

\footnotetext{
${ }^{1}$ Ground Data Systems Engineer, LSE Space at GSOC, DLR

${ }^{2}$ Ground Data Systems Engineer, LSE Space at GSOC, DLR

${ }^{3}$ Ground Data Systems Manager, GSOC, DLR
} 
All these conditions were taken into account when preparing the ground data system and a suitable solution was found by the implementation of SLE online complete transfer services and especially the fine-tuning of some SLE parameters; that will be explained in detail in the following pages.

\section{Initial design for the regular mission scenario}

A satellite mission handover to GSOC was planned by the time the spacecraft was already in orbit for several months. The time to prepare all necessary technical and operational handover tasks was very tight. Handing over an existing mission with a specific design, from one satellite control centre and ground system to another, turned out not to be such an easy task. As it was initially designed, the standard control centre had access to the spacecraft using only one ground station. The ground protocol selected to transfer telecommands and telemetry data from and to the ground station was the Cortex protocol.

The project had strong requirements in terms of telemetry data completeness and the telemetry data rate on the space link was $1 \mathrm{Mbps}$ with all virtual channels to be transferred in real time to the control centre. The initial design allowed the data archiving at the backup control centre collocated with the ground station. This was a huge advantage to achieve the completeness of the offline telemetry delivery since the real time telemetry transfer to the control centre showed frequent data gaps. Unfortunately this option was not available later, due to the fact that more ground stations would be in use and not all of them had backup control centre capabilities.

\section{Ground station network design at GSOC}

In order to quickly develop an operational and reliable ground station network, GSOC selected its own Weilheim ground station with all the existing infrastructure and know-how. It was decided as well to integrate the initial ground station which was already validated and used by the mission for LEOP and routine phase before the handover..

For the Weilheim ground station, prime and backup data links to GSOC used to support all ongoing missions were upgraded to comply with the $1 \mathrm{Mbps}$ requirement dedicated for the new mission. Regarding the ground protocol, the existing SLE infrastructure was employed.

For the initial ground station, since there were other ongoing missions in GSOC that were already making use of it, a functional VPN link through Internet, complying with the $1 \mathrm{Mbps}$ requirements, was already in place. The ground protocol already available was Cortex protocol and, due to the missing SLE provider at the ground station, GSOC decided to continue working with the existing protocol (see Figure 1).

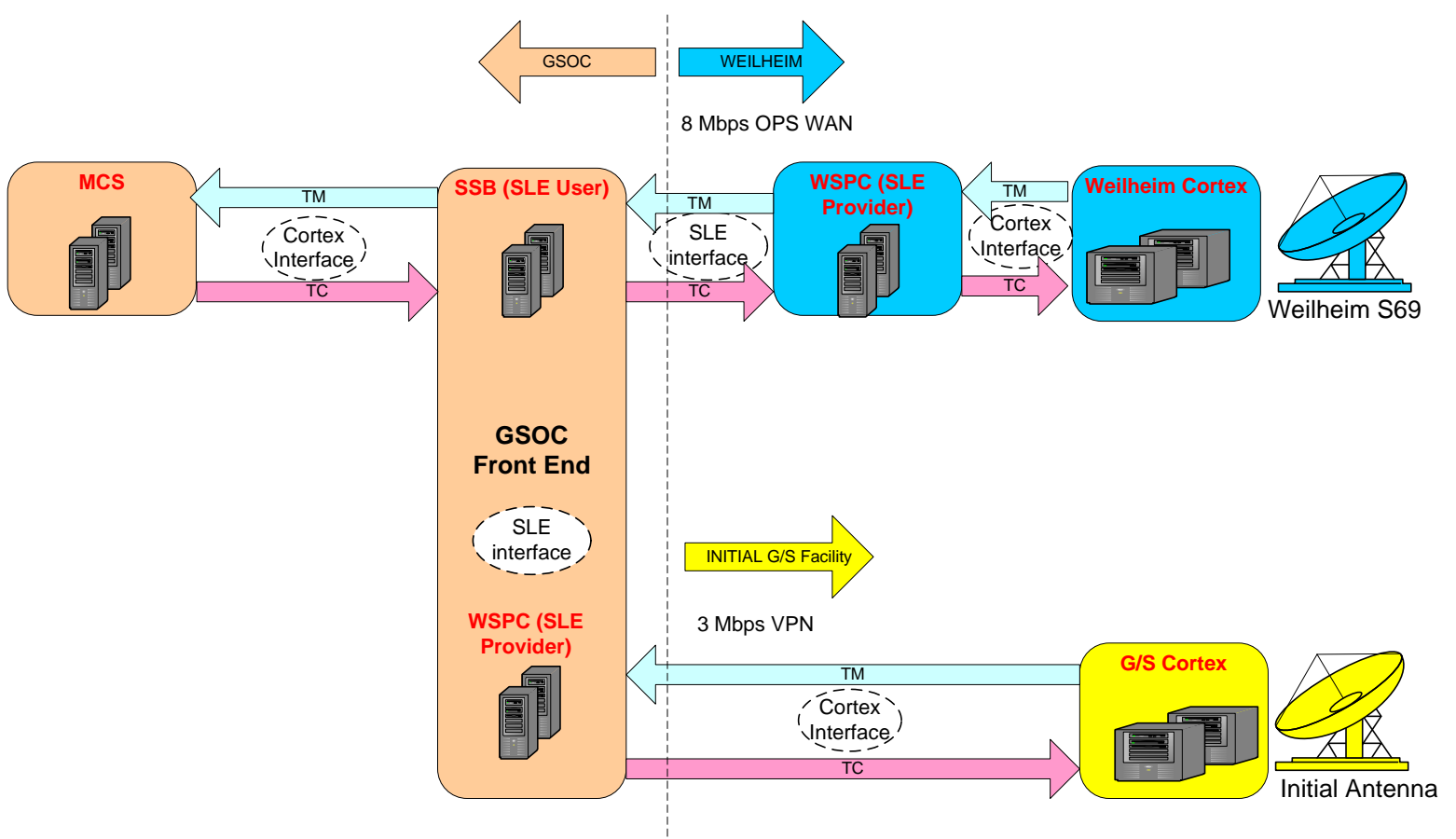

Figure 1. Ground station network at GSOC 


\section{Firsts tests using Weilheim station with SLE}

The first telemetry data flow tests performed with Weilheim encountered data discards while transferring telemetry from ground station to control centre at a rate of 1 Mbps. Here it should be mentioned that the data links to Weilheim provide dedicated bandwidth and are very reliable. It was discovered that the data discards occurred due to the online timely mode configured in the SLE. This SLE transfer mode is the preferred one by most of the projects because fresh telemetry data is needed on the telemetry displays in control room to have continuous updates from the spacecraft, while older data that could not be delivered within a certain time is discarded. When working with online timely delivery mode, the parameter called Transfer Buffer Size of the SLE provider plays a big role, since it indicates the maximum amount of frames that can be stored in the so called transfer buffer. As soon as the transfer buffer is full, the provider will pass all the stored frames to the communications service. The default value of this parameter in our SLE implementation was set to 1 and it turned out to be too small to accommodate the live transfer of data at such a high data rate without TM discards. After several tests, an increase of the value to 150 apparently solved the problem (see Figure 2). With the above SLE settings, Weilheim station was ready to support initial end-to-end telemetry testing including the MCS.

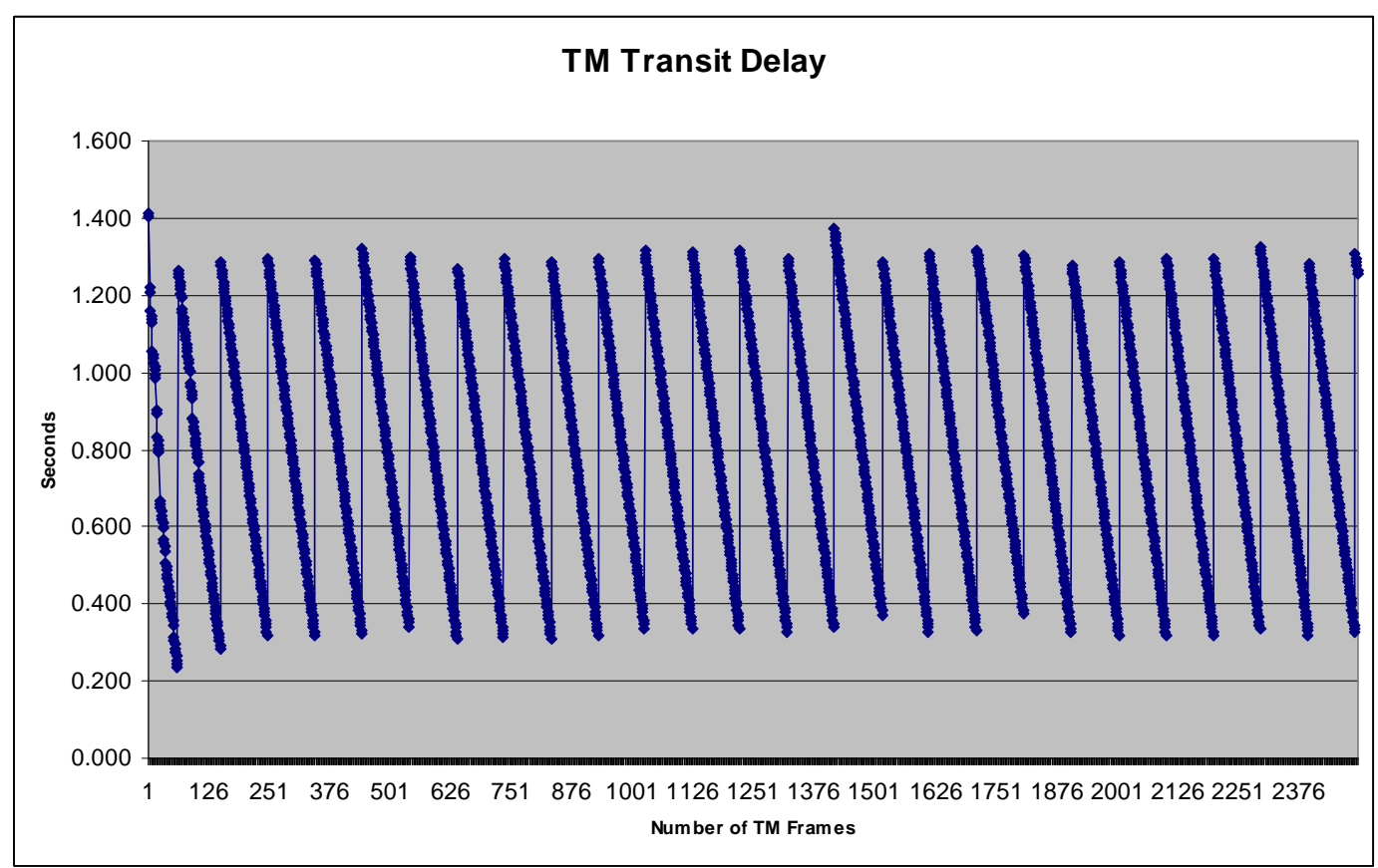

Figure 2. TM transit delay showing how the mechanism of SLE Transfer Buffer Size of 150 TM frames is working.

However, when involving the mission MCS in the end-to-end test we observed that MCS could not deal with the amount of 150 telemetry frames delivered at a time and had issues to process the frames. Decreasing the Transfer Buffer Size from 150 showed repeated data discards at the SLE Provider level and therefore proved that online timely mode would not fit for this mission.

During the integration of the initial ground station, it had been decided to switch to online complete delivery mode as in this mode the service provider shall store all frames acquired from the space link in a buffer called the online frame buffer and therefore data is never discarded. The value of the online frame buffer also had to be adjusted to be sufficiently large to deal with communications service delays, outages, and bandwidth limitations.

Strictly speaking, the transfer buffer is required only in case of online timely delivery mode. However, the transfer buffer still plays a role in the SLE online complete mode, as it allows for "tuning" of the size of the telemetry data units that are passed to the communication equipment (switches, routers and so on). Therefore, in order that the MCS could process the telemetry data error-free we had to decrease the Transfer Buffer Size value down to an optimum value of 5 . With these settings we obtained a reliable error-free telemetry transmission. 


\section{Firsts testing using the initial ground station with Cortex protocol}

As mentioned earlier, the data link available from GSOC to the initial ground station was a VPN through Internet with $3 \mathrm{Mbps}$ setting at both ends, what in principle should have been enough to transfer the $1 \mathrm{Mbps}$ telemetry and the telecommands as well. As shown in the Figure 1, an SLE Provider was installed at the GSOC control centre for the interface with the initial ground station to accommodate all involved ground stations in the same manner. This SLE provider was therefore configured to connect directly to the baseband equipment at the ground station facility using the Cortex protocol. First tests however, were not very positive, as large telemetry data gaps were observed (see Figure 3), calculation of the data gaps being performed by using the Earth Receive Time delta. Each data gap interval consisted of 256 telemetry frames (2.6 seconds in the graph below) and it seemed like a buffer was being cleared each time data gaps occurred.

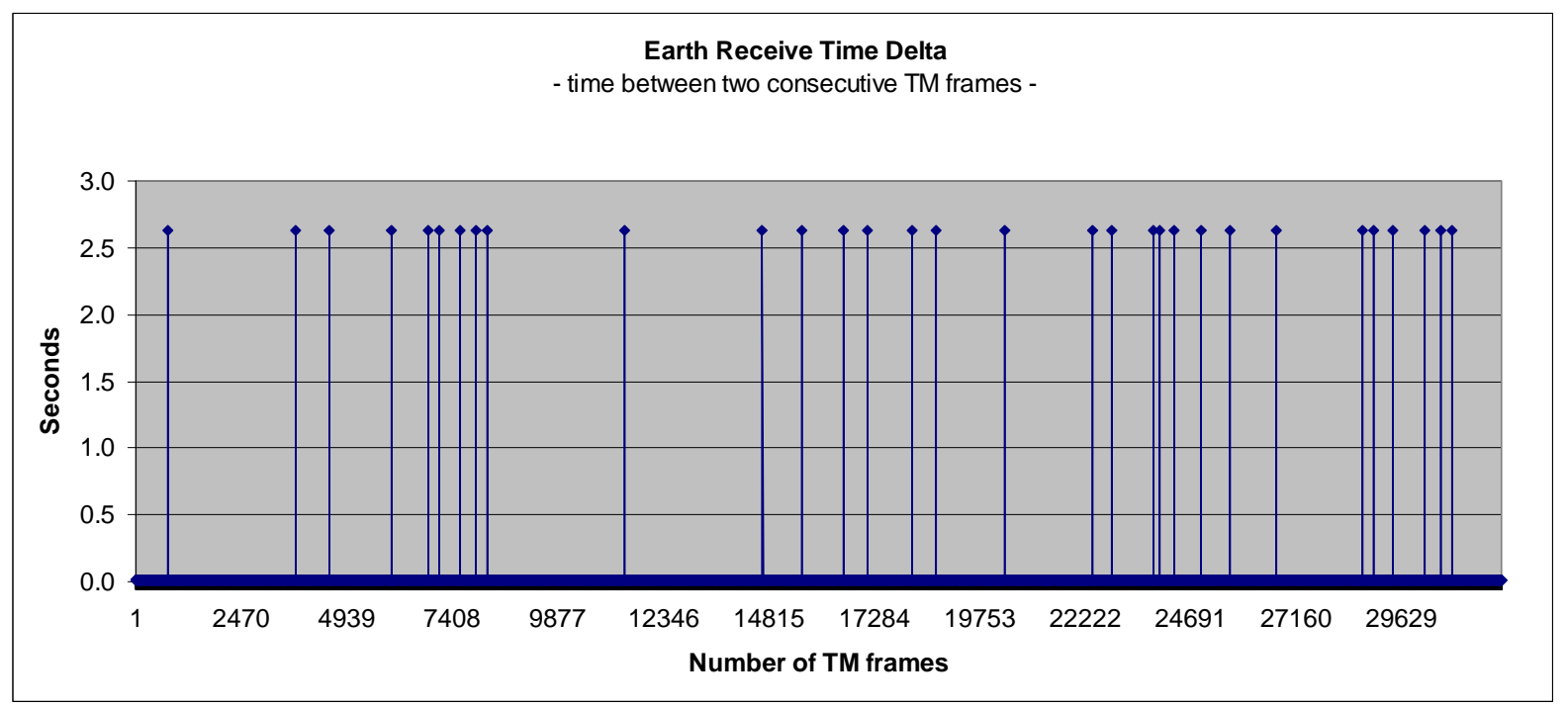

Figure 3. ERT Delta graph showing the 256 lost TM frames at the receiving end

Two questions arose at that point:

Why were we having the data losses? Was the WAN Network over Internet not good enough?

Why 256 frames each time?

Having studied the ground station baseband and protocol documentation, the answer for the second question was found: Cortex has a default value transfer buffer size of 256 frames. It means that, if for some reason the buffer gets full, then all stored telemetry frames are discarded. As a first approach, it was thought that increasing the buffer size could solve the issue. However the maximum value that could be set for the transfer buffer in Cortex was 1024. Configuring this value and repeating the test resulted in the same kind of behaviour, only that this time the data gaps appeared after longer time intervals (see Figure 4 and 5). 


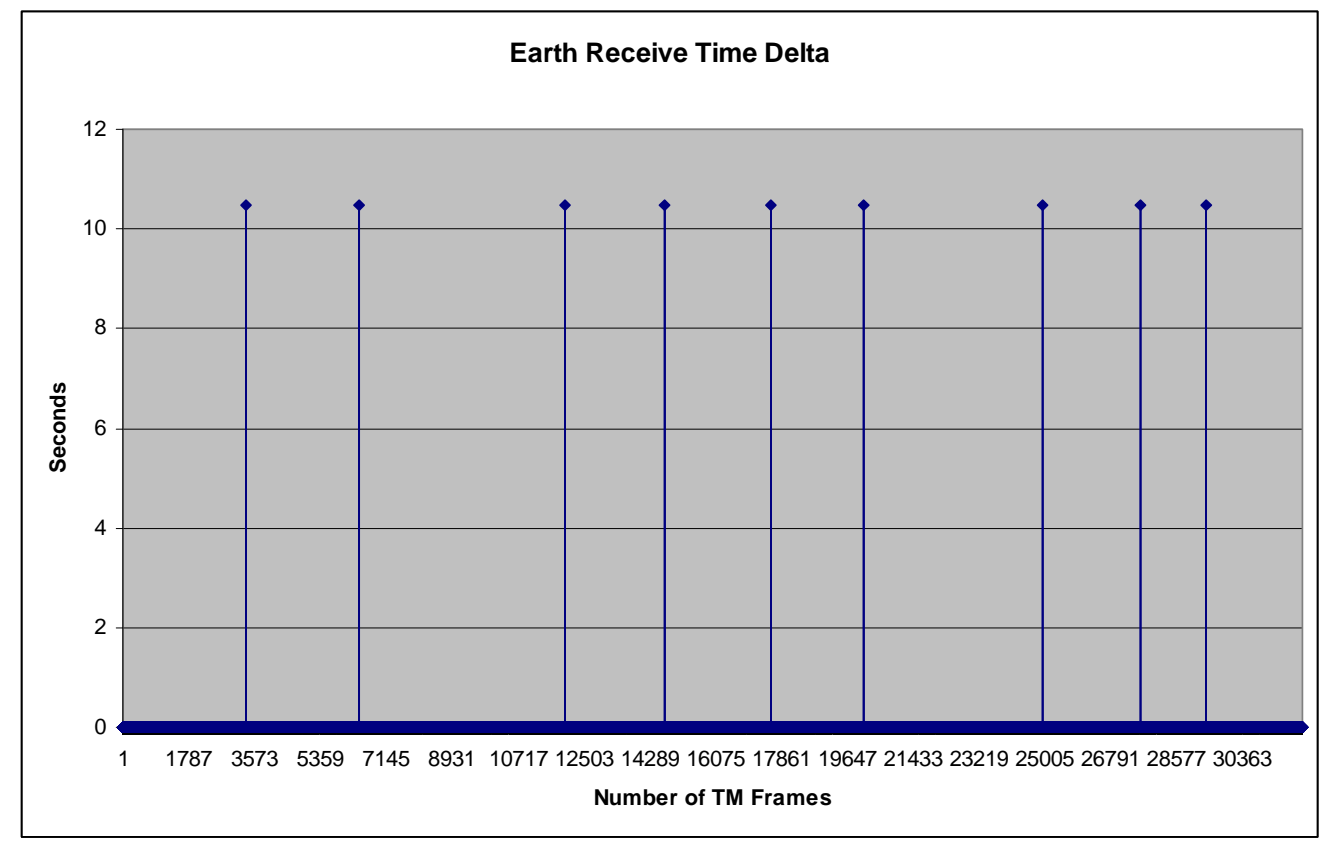

Figure 4. ERT Delta graph showing the 1024 lost TM frames at the receiving end

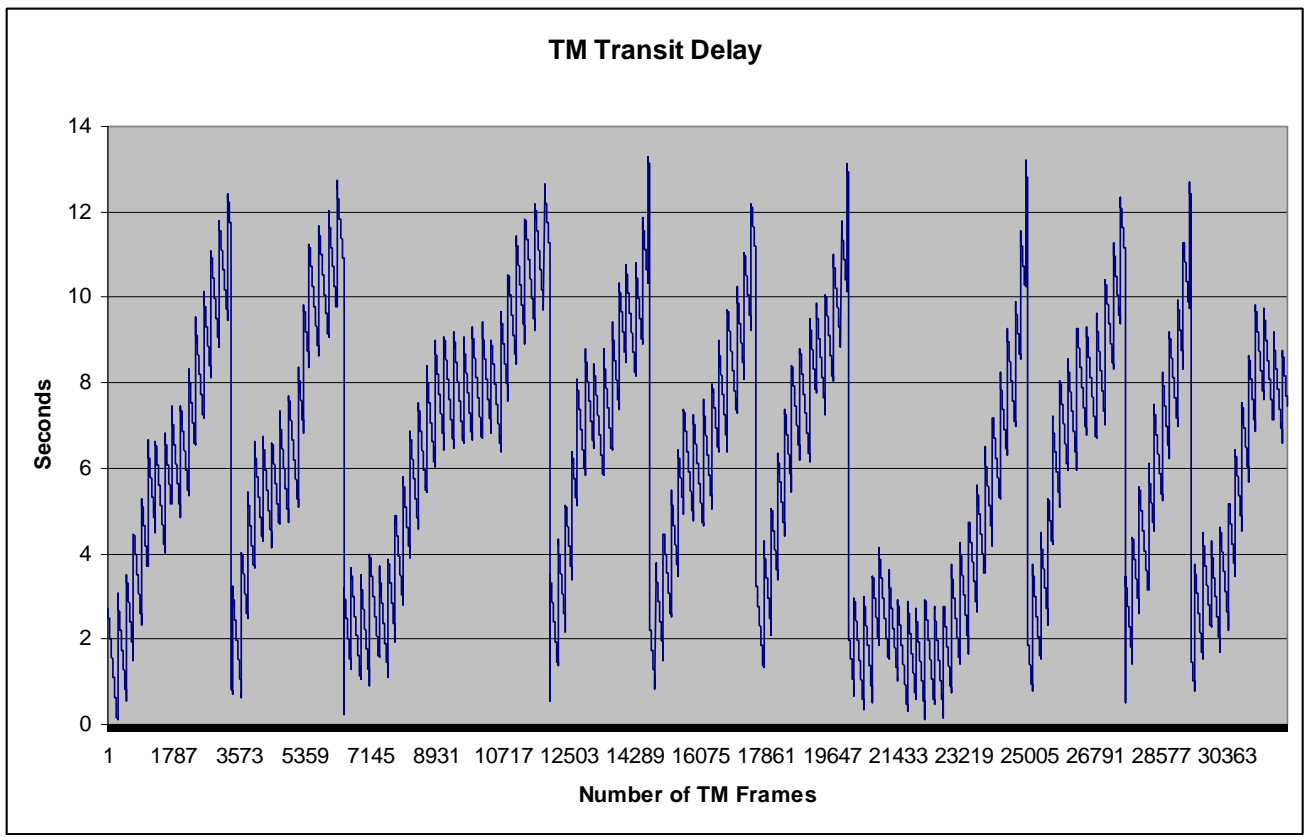

Figure 5. TM transit delay graph showing how the network delay works with the buffer of 1024

To calculate the TM transit delay showed in Figured 5, we calculated the time difference between the Earth Receive Time-stamp information delivered by the Cortex for a given telemetry frame and the time when that telemetry frame was received by the front-end equipment at the control centre. In other words "how much time it takes for a TM frame to travel from the ground station to the control centre”. In Figure 5 we can see how this delay grows from 1.5 seconds, when the first TM frames come right after AOS, to around 12 seconds, when the Cortex buffer has been filled up, and then the Cortex buffer is cleared (see the corresponding spikes in Figure 4). This process is repeated until the end of satellite pass. 


\section{Detailed WAN network tests}

To answer the questions why we had the data losses and if the WAN network was responsible for the data losses, we started looking at the network equipment and measured the bandwidth in real time with test telemetry and parallel transfers of large files using FTP protocol. The first indication from the network analysis was that the bandwidth necessary to transfer the 1 Mbps was enough.

Then we looked at the TCP settings of endpoint equipment at both sides, Cortex at the ground station and SLE provider at control centre. When using TCP to transfer data the two most important factors are the TCP window size and the round trip latency. If the ТСР window size and the round trip latency are known, you can calculate the maximum possible throughput of a data transfer between two hosts, regardless of how much bandwidth you have:

\section{TCP-Window-Size-in-bits / Latency-in-seconds = Bits-per-second-throughput}

In our case, re-configuring the TCP window size to different test values didn't help. However with a default value of 19.6k (default value depending on the operating system, as Cortex NT and Cortex XL differ!) of the TCP window size at the baseband unit and the given round trip latency by the ping command of 70 ms, we could still have a theoretical throughput of 2.2 Mbps.

After all those tests, we managed to convene network experts from both sides and organized a network test by usage and installation on both sides of the commercial IxChariot testing tool developed by Ixia. This method of testing turned out to be very efficient to isolate our problems. The result of the tests was that our VPN link through Internet could support a TCP session up to a maximum of $3.7 \mathrm{Mbps}$, with an average of $1.1 \mathrm{Mbps}$ and sometimes a minimum value of $0.44 \mathrm{Mbps}$ (see Figure 6 ), $0.44 \mathrm{Mbps}$ being less than half of the minimum requirement for online telemetry transfer.

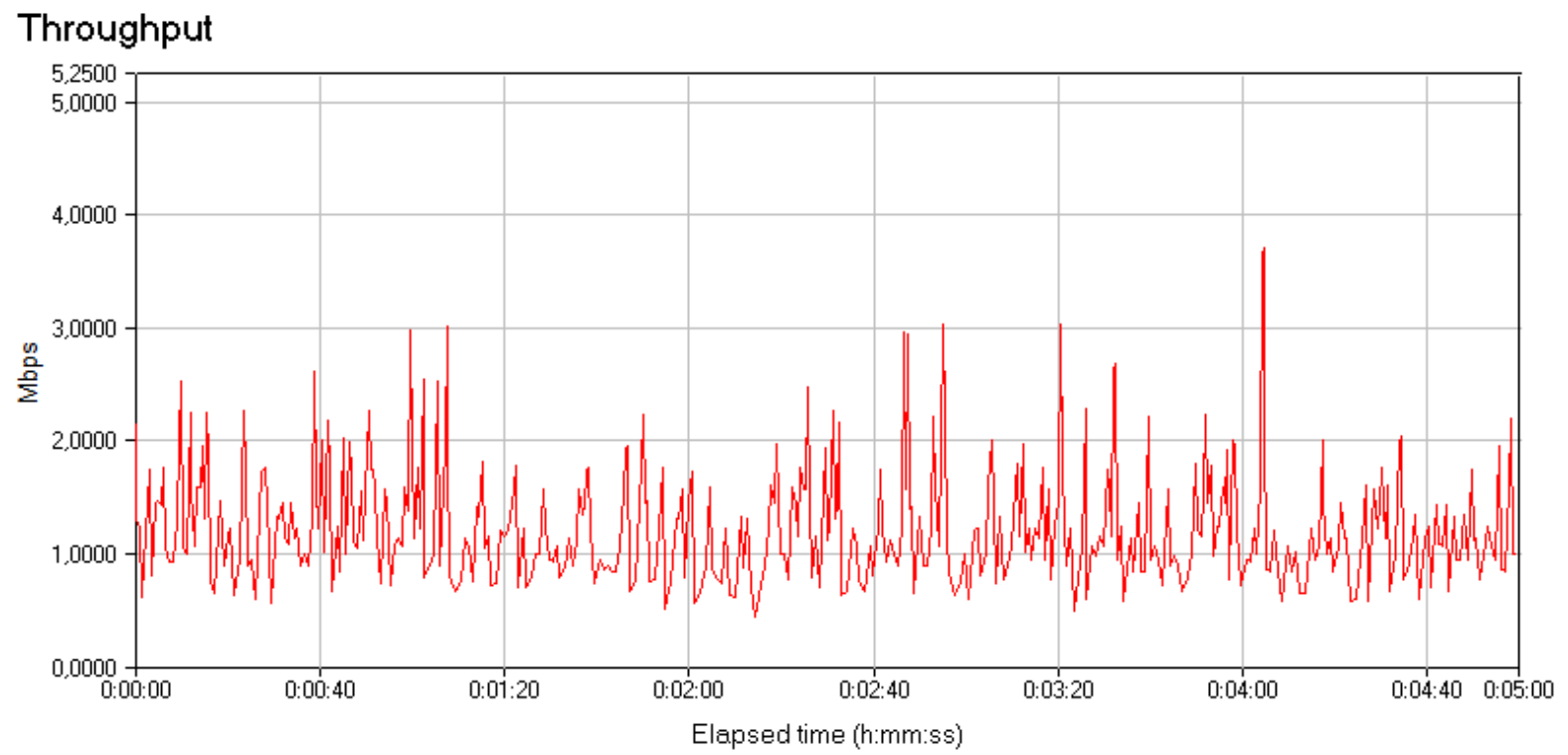

Figure 6. Results of IxChariot network testing of the VPN link

The issue was isolated and it could be described as usage of a data link with non-guaranteed bandwidth sometimes dropping under $1 \mathrm{Mbps}$, and the way the Cortex buffering mechanism works (with a maximum of 1024 TM frames) was not enough to compensate and resulted in data losses.

\section{Implementation of SLE}

Due to the fact that the ordering and implementation of a new reliable data link would exceed the mission timeline requirements, the first thing to do was to discover ways to work with the given unreliable data link. Implementing SLE by installing an SLE provider at the ground station facility close to the baseband equipment 
was the option we started with in the first place. As explained before, the SLE provider has the capability of almost infinite buffering by usage of the online complete delivery mode.

Installation of the SLE provider at the ground station was done in a couple of days and online complete delivery mode showed very positive results for the mission. The huge buffer at the ground station side could compensate all VPN through Internet bandwidth drops under 1 Mbps and most of the satellite passes had a telemetry transit delay pattern as seen in Figure 7. The delay for each frame was below 4 seconds and that was absolutely acceptable for the mission requirements.

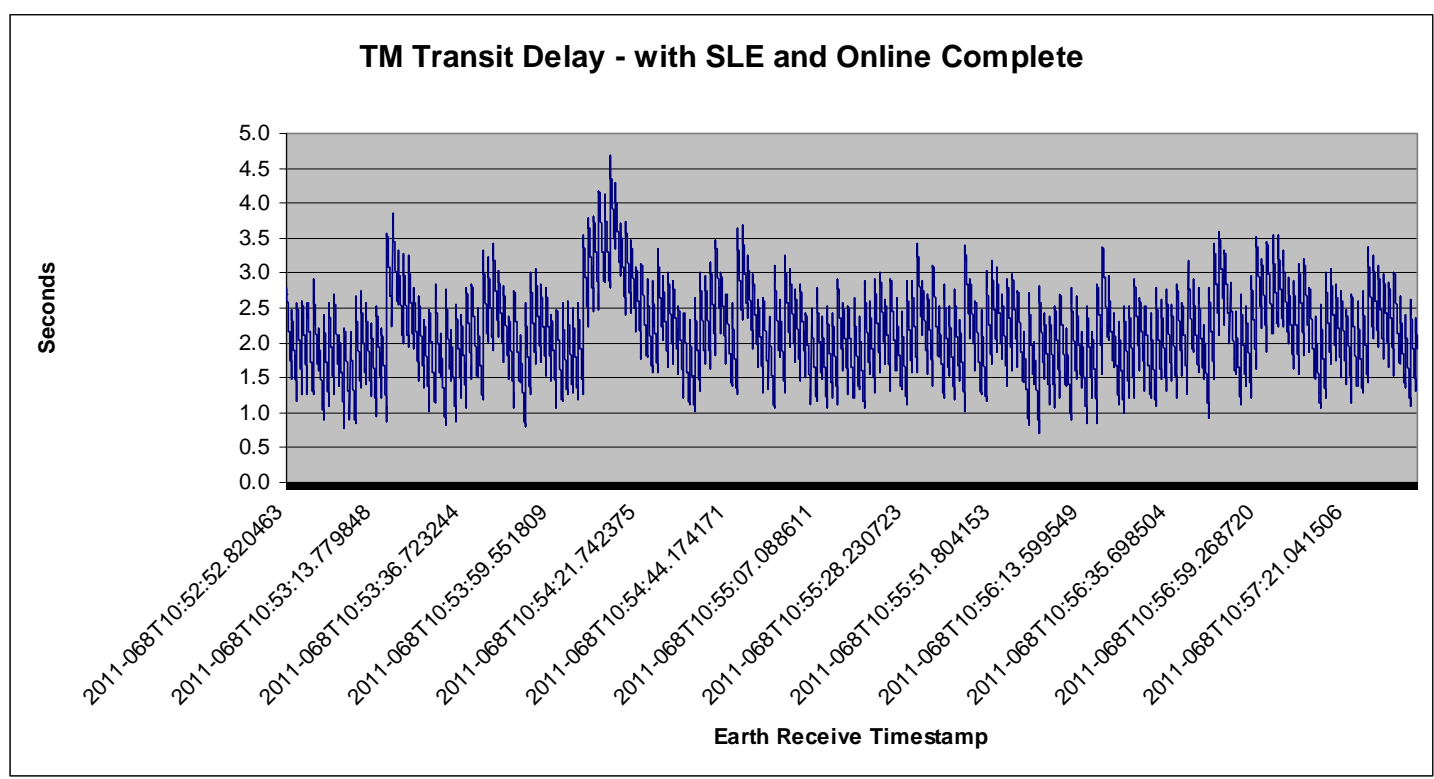

Figure 7. Telemetry transit delay (from G/S to CC) with SLE online complete

However, a few times during mission support, we had such bad VPN through Internet performance resulting in huge delays of telemetry reception at the control centre of sometimes up to 4 minutes! Figure 8 shows such a satellite pass telemetry delay of up to 120 seconds, recovering slowly to 80 seconds after the nominal LOS.

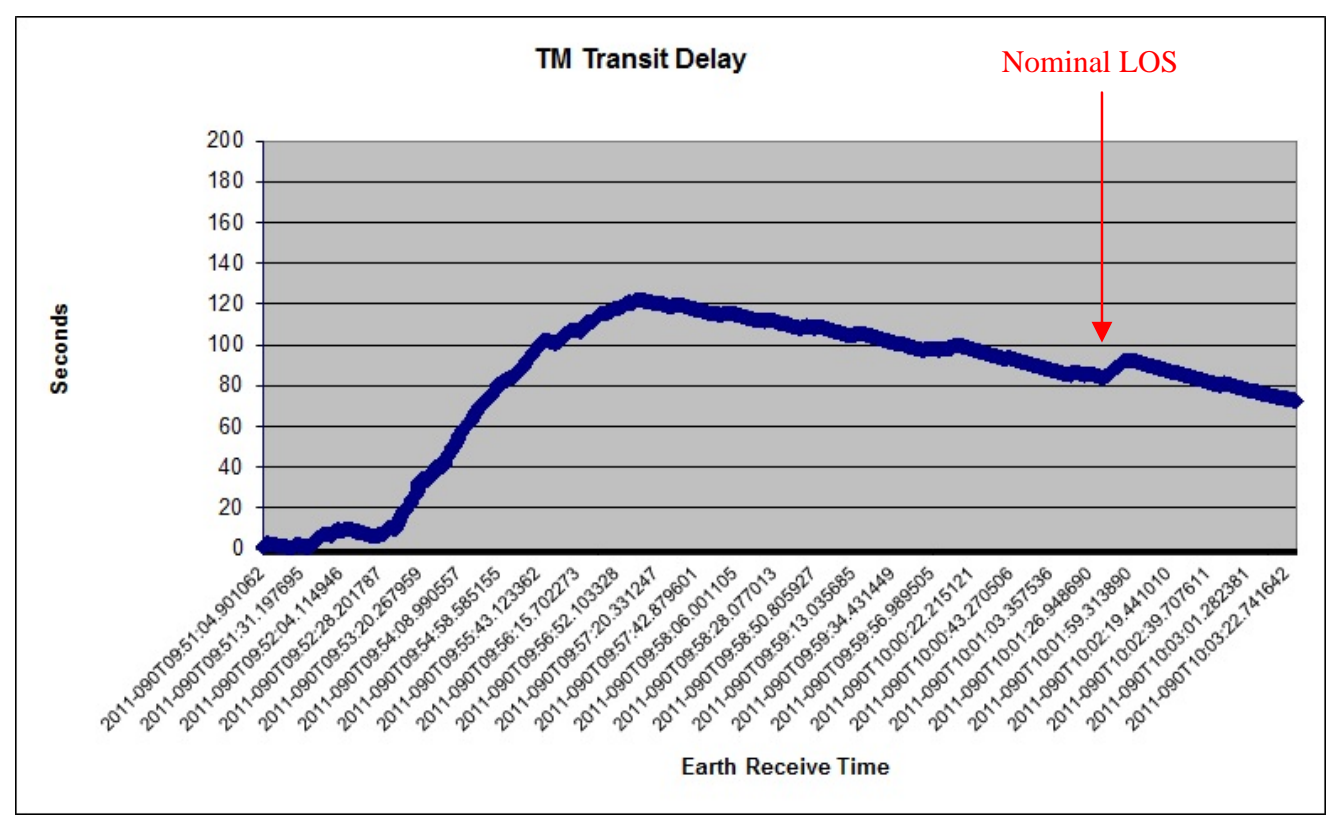

Figure 8. Telemetry transit delay of up to two minutes due to bad performance of the data link 
For those supports, commanding capability of the spacecraft was affected as the reception/radiation confirmation of the telecommands was received too late for MCS to declare the respective CLTU transmission as successful. In such circumstances with limited bandwidth and bottlenecks you may expect that the F-CLTU service will become inactive with the error message " $<F-C L T U>$ SI aborted. Originator local service element, Diagnostic communications failure” as the SLE provider is unable to send the CLTU ACK to the SLE user. We could not find a solution for this, but an efficient operational workaround; in any case, it happened just two or three times during the mission operations.

\section{VPN through Internet vs. MPLS}

Important to mention is that the communication problem described above was not just an isolated case with only one communication link to one ground station, but we have had similar experience having the communication links using VPN partly through Internet to another DLR ground station on another continent. Same experience, same settings of the SLE components and same workarounds were therefore applied to support the mission operations.

However, later on during mission operations, implementing high reliable technologies as MPLS with dedicated bandwidth available fixed all issues and we gathered higher reliability for the overall mission support. The solution using the Internet is very cheap in terms of monthly cost and reliable solutions like MPLS are more expensive, but the quality of service is reflected very well in the reliability / costs ratio.

\section{Conclusion}

After going through the whole integration we have obtained valuable experience to be used for future mission's preparation. CCSDS Space Link Extension protocol proved in this case that there are several options available such as data delivery mode or buffer settings in order to mitigate and compensate unreliable communication links. Such options are missing in other ground protocols, like Cortex protocol, which is probably designed to work properly only in a LAN environment rather than in a WAN environment, at least in case higher data rates are required. As a final recommendation, for satellite mission operations: use the Internet infrastructure with care! 
Appendix A

Acronym List

$\begin{array}{ll}\text { ACK } & \text { Acknowledgement } \\ \text { AOS } & \text { Acquisition Of Signal } \\ \text { ERT } & \text { Earth Receive Time } \\ \text { F-CLTU } & \text { Forward-Command Link Transmission Unit } \\ \text { G/S } & \text { Ground Station } \\ \text { GSOC } & \text { German Space Operation Centre } \\ \text { LAN } & \text { Local Area Network } \\ \text { LEOP } & \text { Launch and Early Orbit Phase } \\ \text { LOS } & \text { Loss Of Signal } \\ \text { MCS } & \text { Mission Control System } \\ \text { MPLS } & \text { Multi-Protocol Level Switching (Cisco protocol) } \\ \text { SLE } & \text { Space Link Extension } \\ \text { SSB } & \text { SLE Switch Board (GSOC's SLE User) } \\ \text { TCP } & \text { Transmission Control Protocol } \\ \text { TM } & \text { Telemetry } \\ \text { VPN } & \text { Virtual Private Network } \\ \text { WAN } & \text { Wide Area Network } \\ \text { WSPC } & \text { Weilheim SLE Provider to Cortex interface (GSOC’s SLE Provider) }\end{array}$

